\title{
Effects of heat release on triple flames
}

\author{
G. R. Ruetsch ${ }^{\text {a) }}$ \\ Center for Turbulence Research, Stanford University, Stanford, California 94305-3030 \\ L. Vervisch \\ LMFN, INSA-Rouen, URA, CNRS 230, CORIA, 76821 Mont-Saint-Aignan Cedex, France
}

A. Liñán

E.T.S. Ingenieros Aeronáuticos, Universidad Politécnica de Madrid, Spain

(Received 6 December 1994; accepted 28 February 1995)

\begin{abstract}
Heat release effects on laminar flame propagation in partially premixed flows are studied. Data for analysis are obtained from direct numerical simulations of a laminar mixing layer with a uniformly approaching velocity field. The structure that evolves under such conditions is a triple flame, which consists of two premixed wings and a trailing diffusion flame. Heat release increases the flame speed over that of the corresponding planar premixed flame. In agreement with previous analytical work, reductions in the mixture fraction gradient also increase the flame speed. The effects of heat release and mixture fraction gradients on flame speed are not independent, however; heat release modifies the effective mixture fraction gradient in front of the flame. For very small mixture fraction gradients, scaling laws that determine the flame speed in terms of the density change are presented. (C) 1995 American Institute of Physics.
\end{abstract}

\section{INTRODUCTION}

Diffusion flames, which exist in reacting flows when fuel and oxidizer are initially separated, play an important role in many engineering flows. In such flows, a significant question concerns how diffusion flames are stabilized. Premixed flames propagate into unburned regions through the diffusion of heat into the premixed reactants, whereas diffusion flames have no such propagation mechanism. It is therefore necessary that diffusion flames be stabilized in partially premixed conditions. Liñan ${ }^{1}$ shows that there are two possibilities for stabilization of diffusion flames in laminar mixing layers: the flame can either be stabilized near the splitter plate, or it can be stabilized farther downstream as a lifted flame. In the former case the flame is anchored in the wake of the splitter plate, where the velocity deficit of the wake and heat conduction to the plate play important roles in stabilization. In the lifted flame these mechanisms are absent, and laminar flame stabilization is achieved through "triple flames;" i.e., a flame that is composed of two premixed flames, one fuel rich and the other lean, and a trailing diffusion flame (see Fig. 1). The two premixed wings provide the ability to propagate, and the diffusion wing provides an anchor for the trailing diffusion flame.

In addition to diffusion flame stabilization, triple flames can also play an important role in the ignition process of nonpremixed systems. Numerical simulations of ignition by Réveillon et al. ${ }^{2}$ show that triple flame propagate along lines of stoichiometric mixture fraction in a weakly stirred mixture of fuel and oxidizer. In addition, Peters ${ }^{3}$ notes that $\mathrm{NO}_{x}$ emissions are likely to be large in such transient cases, and therefore an understanding of triple flames can provide beneficial information concerning pollutant formation.

One of the first observations of triple flames was made by Phillips, ${ }^{4}$ who investigated triple flame propagation in a

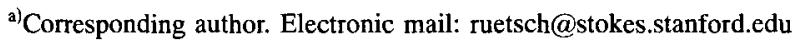

methane mixing layer. More recently, Kioni et al. ${ }^{5}$ have analyzed triple flames, both experimentally and numerically. In their numerical approach, they first developed a model for a triple flame in a counterflow geometry under the assumption of zero heat release, and then solved these equations numerically to obtain the flame structure and speed. There have also been numerous analytical studies of triple flames under various assumptions by Dold, ${ }^{6}$ Dold et al., ${ }^{7}$ and Hartley and Dold. $^{8}$

In this paper we relax the assumption of zero heat release that has been used in many of the previously mentioned analytical studies. McMurtry et al. ${ }^{9}$ studied reacting mixing layers, where the influence of heat release on large-scale structures, entrainment, and other properties was analyzed. However, these simulations were temporal and did not address the issues of stabilization and flame propagation. In order to investigate the role heat release plays in flame propagation under partially premixed conditions, we return to examining the behavior of flames in a laminar flow. We begin by discussing the governing equations used for variable density flows. We then describe the numerical method used for solving this set of equations along with the flow configuration, and follow this by a discussion of the results.

\section{GOVERNING EQUATIONS}

There are several approaches to investigating variable density flows, and in this section we discuss the equations used in our numerical simulations, along with the assumptions and models used in their derivation. Because we are interested in heat release effects, we need to allow for density changes in the flow; for this reason, the fully compressible Navier-Stokes equations are used. If $\rho$ is the total fluid density, $u_{i}$ is the velocity component in the $i$ th direction, and $P$ is the static pressure, these equations can be written as 


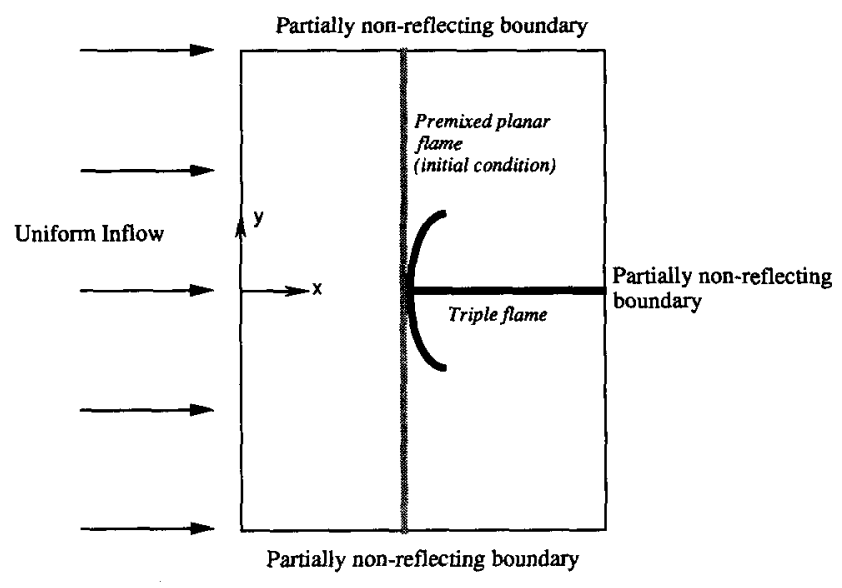

FIG. 1. Computational domain used in the simulations.

$$
\begin{aligned}
& \frac{\partial \rho}{\partial t}+\frac{\partial}{\partial x_{i}}\left(\rho u_{i}\right)=0 \\
& \frac{\partial}{\partial t}\left(\rho u_{i}\right)+\frac{\partial}{\partial x_{j}}\left(\rho u_{i} u_{j}\right)=-\frac{\partial P}{\partial x_{i}}+\frac{\partial \tau_{i j}}{\partial x_{j}}
\end{aligned}
$$

The deviatoric stress tensor $\tau_{i j}$ is given by

$$
\tau_{i j}=\mu\left(\frac{\partial u_{i}}{\partial x_{j}}+\frac{\partial u_{j}}{\partial x_{i}}-\frac{2}{3} \frac{\partial u_{k}}{\partial x_{k}} \delta_{i j}\right)
$$

where $\mu$ is the molecular viscosity, which has a temperature dependence given by

$$
\mu=\mu_{0}\left(\frac{T}{T_{0}}\right)^{a},
$$

with $a=0.76$. The reference state, denoted by the subscript 0 , is taken in the ambient flow.

The energy equation in terms of the total energy density,

$$
\rho e \dot{=}=\frac{1}{2} \rho u_{i}^{2}+\frac{P}{\gamma-1},
$$

is given by

$$
\frac{\partial \rho e}{\partial t}+\frac{\partial}{\partial x_{j}}\left[(\rho e+P) u_{j}\right]=\frac{\partial}{\partial x_{j}}\left(u_{i} \tau_{i j}\right)-\frac{\partial q_{i}}{\partial x_{i}}+Q_{F} \dot{w}_{F} .
$$

Here $\lambda$ is the thermal conductivity, $Q_{F}$ is the heat of reaction per unit mass of fuel, $\dot{w}_{F}$ is the density-based rate of fuel production due to the chemical reaction, $q_{i}$ is the heat flux,

$$
q_{i}=-\lambda \frac{\partial T}{\partial x_{i}}
$$

and $\gamma$ is the ratio of specific heats assumed constant at a value of 1.4 .

In addition to the overall density, we also solve equations for the fuel and oxidizer mass fractions, $Y_{F}$ and $Y_{O}$, defined by

$$
Y_{k}=\frac{\rho_{k}}{\rho}
$$

where $\rho_{k}$ is the partial density of species $k$. In terms of the mass fractions, the conservation equation for individual species becomes

$$
\frac{\partial \rho Y_{k}}{\partial t}+\frac{\partial}{\partial x_{j}}\left(\rho Y_{k} u_{j}\right)=\frac{\partial}{\partial x_{j}}\left(\rho \mathscr{D}_{k} \frac{\partial Y_{k}}{\partial x_{j}}\right)+\dot{w}_{k},
$$

with $\mathscr{\mathscr { V }}_{k}$ and $\dot{w}_{k}$ representing the mass diffusivity and the source term of species $k$ due to the chemical reaction, respectively. The thermal conductivity and the mass diffusivities are temperature dependent in such a way as to maintain constant Lewis and Prandtl numbers,

$$
\mathrm{Le}_{k}=\frac{\lambda}{\rho \mathscr{D}_{k} c_{p}} ; \quad \operatorname{Pr}=\frac{\mu c_{p}}{\lambda},
$$

throughout the flow at all times. In order to close this system of equations an equation of state is required: the perfect gas law,

$$
P=\rho R T,
$$

will be used.

The chemical scheme is represented by a one-step global reaction between fuel and oxidizer:

$$
F+O \rightarrow P,
$$

where we have assumed unity stoichiometric coefficients for simplicity. The reaction rate has the Arrhenius form:

$$
\dot{w}=K \rho Y_{F} \rho Y_{O} \exp \left(-\frac{T_{\mathrm{ac}}}{T}\right),
$$

where $T_{\mathrm{ac}}$ is the activation temperature and $K$ is the preexponential factor. Following Williams, ${ }^{10}$ we can write this reaction rate in terms of the reduced pre-exponential factor, heat release parameter, Zel'dovich number, and reduced temperature:

$$
\begin{aligned}
& \Lambda=K \exp \left(\frac{-\beta}{\alpha}\right) ; \quad \alpha=\frac{T_{f}-T_{0}}{T_{f}} ; \\
& \beta=\frac{\alpha T_{\mathrm{ac}}}{T_{f}} ; \quad \theta=\frac{T-T_{0}}{T_{f}-T_{0}},
\end{aligned}
$$

with $T_{f}$ being the adiabatic flame temperature, resulting in the following expression for the reaction rate:

$$
\dot{w}=\Lambda \rho Y_{F} \rho Y_{O} \exp \left(-\frac{\beta(1-\theta)}{1-\alpha(1-\theta)}\right) \text {. }
$$

For our particular chemical scheme, we assume equal molecular weights of fuel and oxidizer, so that the consumption of both fuel and oxidizer is given by the reaction rate, such that $\dot{w}_{F}=\dot{w}_{O}=-\dot{w}$.

\section{A. Numerical simulation and flow configuration}

The above set of equations are solved in the twodimensional domain depicted in Fig. 1. Spatial derivatives are approximated by the sixth-order compact difference algorithm of Lele, ${ }^{11}$ and the equations are integrated in time with a third-order Runge-Kutta scheme. Boundary conditions are specified using the Navier-Stokes characteristic boundary condition method of Poinsot and Lele, ${ }^{12}$ where an inflow boundary condition is used on the left, and near- 
perfect reflective boundary conditions, required to avoid pressure drift, are used at the outflow and sides. Although the inflow conditions are prescribed, their values can be changed during the simulation. Within this domain we initialize the flow with a planar premixed flame, where the mixture fraction, defined as

$$
Z=\frac{1+Y_{F}-Y_{O}}{2}
$$

is everywhere equal to its stoichiometric value, $Z_{s}=0.5$. The incoming flow is uniform and set equal to the premixed laminar flame speed, $S_{L}^{0}$. Also associated with the flame is the premixed flame thickness, $\delta_{L}^{0}$. In this study we hold the Zel'dovich number constant at $\beta=8$ and use heat release parameters of $\alpha=0.5, \frac{2}{3}, 0.75$, and 0.8 .

After the flow and flame are initialized, the mixture fraction is varied at the inlet from its uniform stoichiometric value to a tanh profile varying from zero to unity. We characterize the thickness of this mixing layer by the slope of the profile at stoichiometric conditions and the overall change in mixture fraction, which gives

$$
\delta_{M}(x)=\Delta Z\left(\left.\frac{\partial Z}{\partial y}\right|_{y=0}\right)^{-1}=\left(\left.\frac{\partial Z}{\partial y}\right|_{y=0}\right)^{-1}
$$

where the last equality is obtained since the overall change in the mixture fraction is unity. It is important to realize that this measure does not remain constant along the stoichiometric line and is a function of $x$. We can form a Damköhler number, which is a ratio of the physical to chemical time scales, using the mixing thickness and the planar flame thickness. Since the characteristic velocity for both chemical and physical processes is the same, the Damköhler number is defined as

$$
D(x)=\frac{\tau_{p}}{\tau_{c}}=\frac{\delta_{M}(x)}{\delta_{L}^{0}}
$$

which can be thought of as a dimensionless mixing thickness. We are concerned with values of the dimensionless mixing thickness at two locations in the flow. The value at the inlet is designated by $D_{0}$ and is a parameter of the simulation, and the value at the location of the maximum reaction rate is designated by $D_{\mathrm{TF}}$ and is more physically relevant to the flame's behavior.

An example of a flame's response to a variable mixture fraction is shown in Fig. 2, with the uniform flow approaching from the left. After the mixture fraction gradient reaches the flame surface, only the centerline is exposed to the stoichiometric mixture fraction; the mixture is fuel rich above and fuel lean below the centerline. These regions of nonunity equivalence ratio burn less fuel, resulting in reduced reaction rates and local flame speeds. Thus, the planar flame speed and reaction rate are maintained only at the centerline. The excess fuel and oxidizer on either side of the centerline then combine along the stoichiometric surface behind the premixed flame and burn in a trailing diffusion flame. Thus, the "triple" flame refers to the fuel-rich premixed flame, the fuel-lean premixed flame, and the trailing diffusion flame.

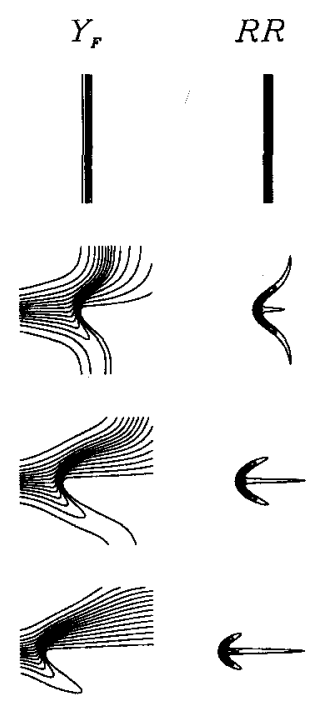

FIG. 2. Response of a planar premixed flame to a mixture fraction gradient. The first row in the time sequence shows the premixed flame in a steadystate situation. As the mixture fraction gradient reaches the flame, the structure and velocity of the flame change. The rows correspond to times of $t S_{L}^{0} / \delta_{L}^{0}=0,11.6,16.3$, and 24.7. The plot size is $18.7 \delta_{L}^{0}$ on each side. Parameters for this data are $\alpha=0.75, S_{L}^{0} \delta_{L}^{0} / \nu=5.4$, and $\Lambda \delta_{L}^{0} \rho_{0} / S_{L}^{0}=6609$. Fifteen contour levels are drawn at equal intervals covering each variable's range.

In addition to the change in structure that occurs when the planar premixed flame is subjected to a mixture fraction gradient, the propagation velocity of the flame increases, as observed in Fig. 2. In order to study the triple flame in further detail, a method of stabilizing the flame in the computational domain is needed. The method of flame stabilization used in this study requires the evaluation of the relative progression velocity of isoscalar surfaces. This quantity is determined by equating the transport equation for a scalar variable $Y$ :

$$
\rho \frac{D Y}{D t}=\frac{\partial}{\partial x_{i}}\left(\rho \mathscr{D} \frac{\partial Y}{\partial x_{i}}\right)+\dot{w}_{Y},
$$

with the Hamilton-Jacobi equation for the scalar field (Kerstein et al. ${ }^{13}$ ):

$$
\rho \frac{D Y}{D t}=\rho V|\nabla Y| \text {. }
$$

Solving for the relative progression velocity of the isoscalar surface, $V$, we obtain:

$$
V=\frac{1}{\rho|\nabla Y|} \frac{\partial}{\partial x_{i}}\left(\rho \mathscr{D} \frac{\partial Y}{\partial x_{i}}\right)+\frac{1}{\rho|\nabla Y|} \dot{w}_{Y} .
$$

This relation is evaluated on the centerline in the preheat zone and subtracted from the local fluid velocity, giving the correction to be applied at the inlet. If one were to apply this correction at the inlet alone, then changes to the flame would only occur after the convective time required to reach the flame, which is both time consuming and can also introduce stability problems. A more efficient method is to apply the correction to all points in the flow, as a Galilean transformation, such that the steady-state situation is quickly reached. 
TABLE I. Simulation parameters and results for stabilized triple flames. The scaled mixing thicknesses $D_{0}$ and $D_{\mathrm{TF}}$ refer to centerline values at the inlet and location of maximum reaction rate, respectively. In addition to these parameters, all simulations have $\beta=8.0, \mathrm{Le}=1$, and $\operatorname{Pr}=0.75$.

\begin{tabular}{lllrrrr}
\hline \hline Simulation & $\alpha$ & $\operatorname{Re}=S_{L}^{0} \delta_{L}^{0} / \nu_{0}$ & $\Lambda \delta_{L}^{0} \rho_{0} / S_{L}^{0}$ & \multicolumn{1}{c}{$D_{0}$} & \multicolumn{1}{c}{$D_{\mathrm{TF}}$} & $S_{\mathrm{TF}} / S_{L}^{0}$ \\
\hline I & 0.75 & 5.4 & 6880 & 0.9 & 8.3 & 1.23 \\
II & 0.75 & 5.4 & 6880 & 1.8 & 8.9 & 1.26 \\
III & 0.75 & 5.4 & 6880 & 3.7 & 11.1 & 1.33 \\
IV & 0.75 & 5.4 & 6880 & 5.5 & 13.7 & 1.39 \\
V & 0.75 & 5.4 & 6880 & 9.2 & 19.7 & 1.46 \\
VI & 0.75 & 5.4 & 6880 & 18.3 & 34.0 & 1.51 \\
VII & 0.5 & 3.9 & 1590 & 25.7 & 35.8 & 1.17 \\
VIII & 0.66 & 4.7 & 3850 & 21.3 & 35.0 & 1.36 \\
IX & 0.8 & 6.1 & 10670 & 16.4 & 33.4 & 1.61 \\
\hline \hline
\end{tabular}

Some care must be taken in choosing the size of the computational domain. Since the triple flame redirects the flow laterally, the top and bottom boundaries must be moved far enough away from the flame so that the viscous boundary conditions do not affect the flame speed. To avoid this problem, an unevenly spaced grid is used in the lateral direction with points clustered about the stoichiometric line, so that the lateral boundaries can be moved far away from the flame. In addition, all results presented here were run on different grids to check that the size of the computational domain does not affect the flame speed. Parameters and primary results for the stabilized triple flames are shown in Table I.

\section{RESULTS}

To begin the investigation of heat release effects on triple flames, we first describe some general characteristics of triple flames. The structure of triple flames was briefly described in the time sequence of Fig. 2, where the flame propagates relative to the inflow. Figure 3 shows similar contours, in addition to the temperature and density fields, for a triple flame stabilized in the computational domain. The ef-

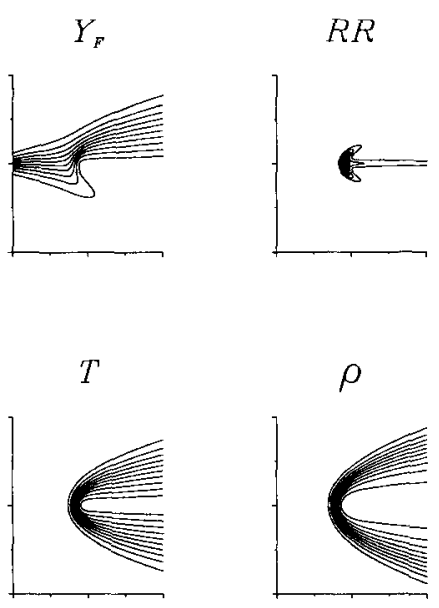

FIG. 3. Contours of mass fraction, reaction rate, temperature, and density for a stabilized triple flame. Results shown are from simulation II in Table I. The domain in the horizontal and vertical directions is $14.7 \delta_{L}^{0}$ and $17.7 \delta_{L}^{0}$. Ten contours are shown with equal increments spanning the entire range of each variable.
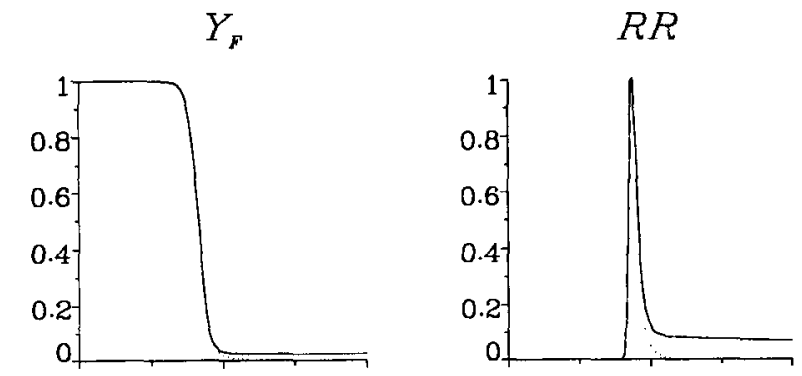

$\mathbf{x}$

$\mathrm{X}$

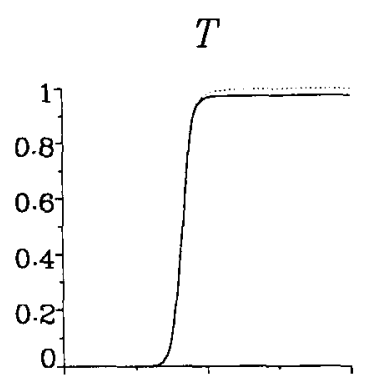

$\mathbf{X}$

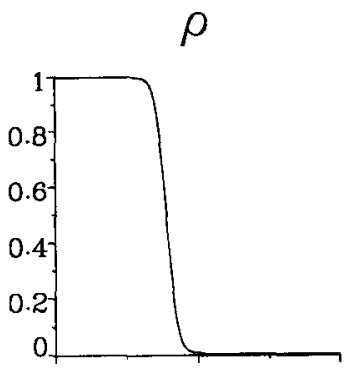

$\mathbf{x}$
FIG. 4. Profiles of various quantities in a one-dimensional flame $(\cdots)$ and along the centerline of the triple flame (-). Lateral heat conduction reduces the temperature of the triple flame, and diffusion of fuel and oxidizer increase the reaction rate behind the premixed flame. The triple flame data are from simulation II. The horizontal axis is of length $14.7 \delta_{L}^{0}$.

fects of heat release are clearly seen in the temperature and density plots; the temperature rises and density decreases behind the premixed wings of the flame, with the lateral variations due to the nonuniform mixture fraction.

Lateral diffusion of temperature and species plays an important role in triple flames. Behind the premixed wings of the flame, heat is conducted away from the stoichiometric line. This conduction is important because, unlike onedimensional flames with an infinite heat reservoir in the burned regions, triple flames are more likely to be quenched if enough heat is conducted away from the premixed flames. Lateral diffusion of the species is also important, and is responsible for the diffusion wing of the flame.

Another way to determine the role of lateral diffusion is to compare one-dimensional profiles of different quantities along the stoichiometric line of the triple flame with those of the one-dimensional flame used to initialize the simulations. These plots are presented in Fig. 4, where the quantities are scaled by their minimum and maximum values of the onedimensional flame. These quantities coincide up to, and somewhat behind, the maximum reaction rate. Behind the premixed reaction zone we observe differences between the one-dimensional and triple-flame cases, although the density profiles remain equal. A small drop in temperature and an increase in fuel (and also oxidizer) mass fraction occur in the triple flame. The change in reaction rate is dominated by the 

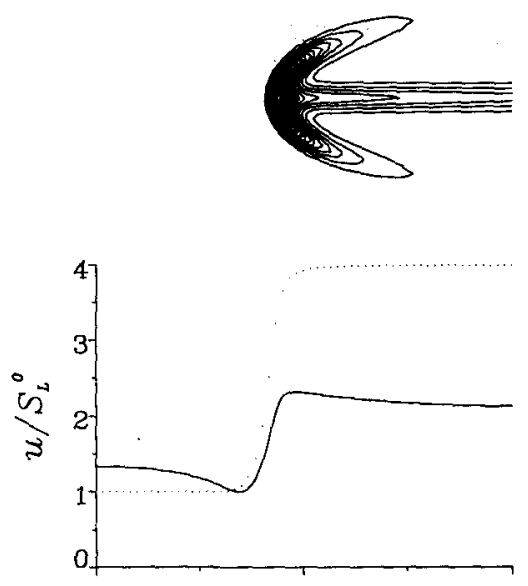

FIG. 5. Contour lines of the reaction rate along with the horizontal velocity component at the stoichiometric or symmetry line for a stabilized triple flame (-) and planar premixed flame $(\cdots)$. For the triple flame the velocity reaches a minimum just in front of the flame, which is close the the premixed planar flame velocity. Upstream of the flame, however, the velocity is larger. The data in this figure are from simulation III, and 30 equally spaced contours are shown in the upper figure.

increased mass fractions of the reactants, and consequently an increase in the reaction rate is observed behind the flame. This reaction rate corresponds to the burning in the trailing diffusion flame, and is a sizable percentage of the maximum reaction rate. These differences between the one-dimensional profiles and profiles through the stoichiometric line in the triple flame become larger as we increase the mixture fraction gradient.

Up to this point we have not included the velocity in our analysis. We have postponed this until the next section, since the velocity field of the triple flame is quite different from that of the planar flame, and requires a detailed investigation.

\section{A. Effects of heat release on flame propagation}

We now turn our attention to studying the effect of heat release on the triple flame, and, in particular, how this affects the propagation velocity. The analytical work of Dold ${ }^{6}$ and Hartley and Dold ${ }^{8}$ provide estimates of the triple-flame speed for weak $(\beta \partial Z / \partial y \rightarrow 0)$ and moderate $[\beta \partial Z / \partial y \sim O(1)]$ values of the mixture fraction gradient under the assumption of zero heat release. They find that the flame speed is greatest for zero mixture fraction gradient, corresponding to a planar flame, and then decreases as the mixture fraction gradient increases. This is in contrast to the change in flame speed we observe from the time sequence in Fig. 2. The discrepancy lies in the assumptions concerning heat release. To investigate this further, we examine the velocity field along the centerline of the triple flame in Fig. 5 . In addition to the rise in velocity through the flame, the horizontal velocity component reaches a minimum before the flame. The velocity at this minimum is close to the planar laminar flame speed, and far upstream the velocity is larger. Therefore it is necessary to distinguish these two velocities. The local flame speed determines the rate of chemical reaction, whereas the upstream or far-field flame speed is identified with the propagation of the entire structure, $U_{F}$.

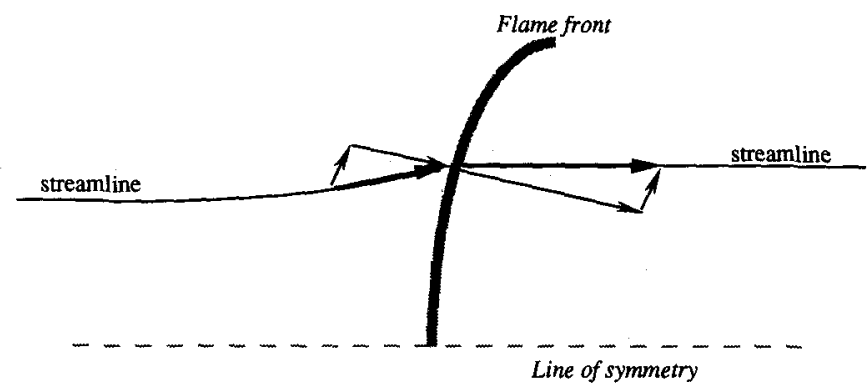

FIG. 6. The mechanism responsible for increased flame speeds. Due to heat release the normal velocity across the flame is increased, whereas the tangential component remains unchanged. This redirection of flow toward the centerline causes the streamlines to diverge in front of the flame, resulting in a decrease in the flow velocity in front of the flame.

The mechanism responsible for this velocity difference can be seen in the sketch of Fig. 6, which illustrates the velocity vectors before and after they pass through the flame surface. In cases where there is heat release, the component of the velocity perpendicular to the flame increases across the surface, whereas the tangential component remains unchanged. The jump in the perpendicular velocity component bends the velocity vector toward the centerline. This redirection of the flow is accommodated by the divergence of the streamlines ahead of the flame, resulting in the decrease of the velocity observed in Fig. 5. Since the local flame speed along the stoichiometric line is approximately $S_{L}^{0}$, the flame can be stabilized only if the flow speed at this point remains at this value, which requires an increase in the upstream velocity. Note that in the absence of heat release, there is no flow redirection across the flame, and therefore the far-field and local flame speeds are equal.

\section{B. Effect of mixture fraction gradients}

In their analyses, Dold ${ }^{6}$ and Hartley and Dold ${ }^{8}$ observed a large effect of the mixture fraction gradient on the tripleflame propagation. They observed a decrease in the flame speed as the mixture fraction gradient increases, or, equivalently, the mixing thickness decreases, due to the effects of flame curvature. Thus, for zero heat release the planar premixed flame is an upper limit for the flame speed.

For cases with heat release, locally these same arguments still apply, however, the far-field flame speed is much more affected by heat release than by flame curvature. This is depicted in Fig. 7, where the far-field flame speed, $U_{F} / S_{L}^{0}$, and the local flame speeds are plotted versus the nondimensional mixing thickness, or Damköhler number. Here we see that, in agreement with the zero heat release analysis, the local flame speeds remain on the order of $S_{L}^{0}$, decreasing slightly below this value for small values of the mixing thickness. Also plotted in this figure is the difference between the local and far-field velocities. This difference eliminates the change in local conditions, and is therefore a true measure of the effect of heat release. As one can see from Fig. 7, this difference increases as the mixing thickness becomes larger. 


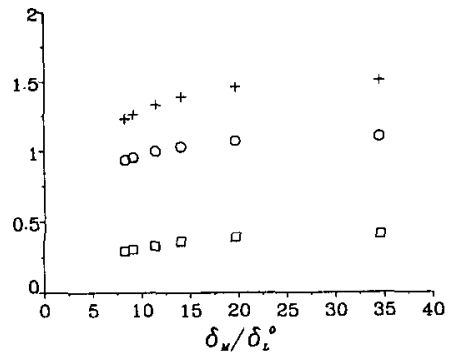

FIG. 7. Farfield flame speed $(+)$, local flame speed $(O)$, and their differences $(\square)$ as a function of the local mixing thickness. All flame speeds are normalized by $S_{L}^{0}$, and data are from simulations I-VI.

The reason for the increased effect of heat release as the mixing thickness becomes larger can be explained by Fig. 8 . In Fig. 8 we plot the streamfunction through two flames with different mixing thicknesses. Since the maximum reaction rate is at the same streamwise location, we can superpose the two streamline patterns to determine how the flow redirection differs in these two cases. At streamwise locations near the maximum reaction rate and close to the stoichiometric line the two cases are similar. As we progress farther downstream, the case with the smaller mixing thickness spreads more laterally due to the greater burning in the diffusion flame. As we move laterally to regions farther from stoichiometric conditions, the deflections of the streamlines away from the stoichiometric line become greater for the larger mixing thickness case. Recall that the mechanism for increased far-field flame speed relies on the acceleration of the normal velocity component through the flame. The local velocity jump across the flame is strongly related to the local reaction rate, which is, in turn, affected by the local mixture fraction. Thus, the distribution of the reaction rate along the premixed wings becomes an important characteristic, and is the reason for the different streamline pattern farther from stoichiometric conditions. For small mixing thicknesses, the reaction rate drops off quickly as one moves away from
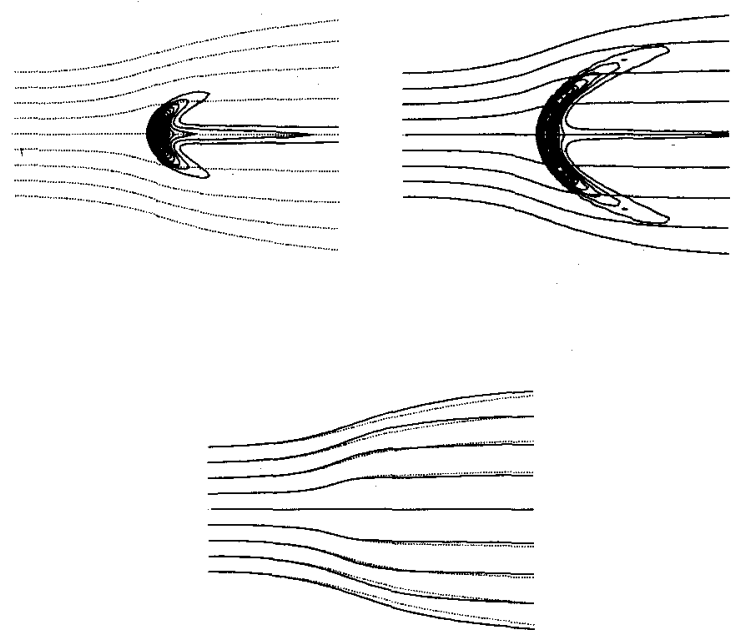

FIG. 8. Streamlines for flames with small (left, $\cdots$, simulation II) and large (right, -, simulation $\mathrm{V}$ ) mixing thicknesses. The streamlines are superposed beneath.

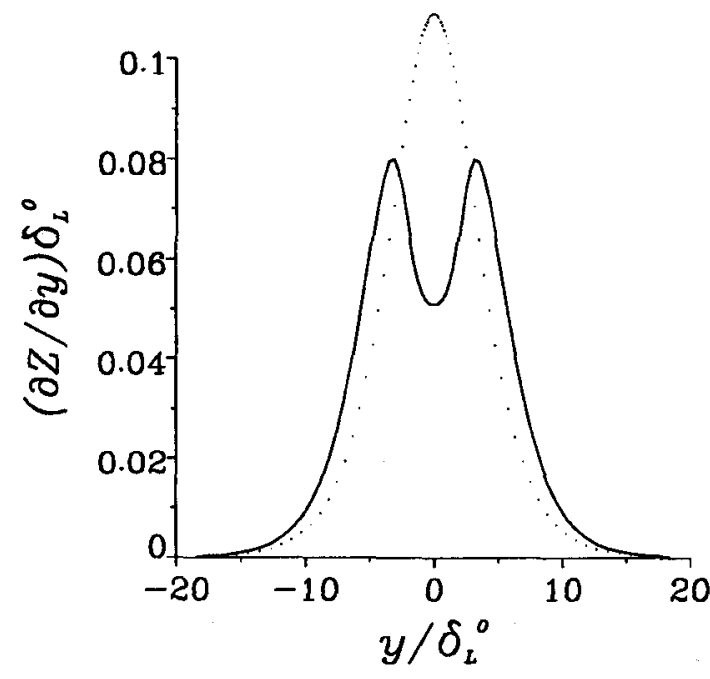

FIG. 9. The vertical mixture fraction gradient profile at the inlet $(\cdots)$ and on the vertical line passing through the maximum reaction rate (-). Data are from simulation V. At $y / \delta_{L}^{0}=0$ we recover $1 / D_{\mathrm{TF}}$ from Table I.

stoichiometric conditions. For larger mixing thicknesses the reaction rate remains stronger as one moves along the premixed wings, and thus the redirection in the flow is more pronounced.

\section{Small mixing thicknesses and resistance to quenching}

For small values of the mixing thickness one might expect quenching to occur. Quenching would result from the lateral conduction of heat away from the flame, which is intensified as the mixing thickness decreases. In previous analytical work, ${ }^{6,8}$ however, quenching was not observed. Under the assumption of zero heat release, quenching was present only when the flame was subjected to an external strain field (Dold et $a l^{7}$ ). Kioni et al. ${ }^{5}$ have simulated triple flames under external strain and have found negative propagation velocities, or an extinction front, to occur when the mixing thickness becomes small enough, once again under the assumption of zero heat release. In our present study, therefore, we do not expect quenching to occur since no external strain is present. Furthermore, in cases with heat release the resistance to quenching is enhanced. This added resistance to quenching occurs because the flame-generated strain field that reduces the horizontal velocity also reduces the effective mixture fraction gradient in front of the flame. Therefore, heat release limits how small the effective Damköhler number can become. This reduction in the local mixture fraction gradient is observed in Fig. 9. Here the mixture fraction gradient along vertical slices is shown at the inlet and on a slice through the maximum reaction rate. The mixture fraction gradient is reduced by diffusion, but near stoichiometric conditions the effect of the heat releaseinduced strain on the mixture fraction gradient is dominant.

\section{Large mixing thicknesses and scaling laws}

We have seen that for the range of mixing thicknesses considered in this study, as the mixing thickness is increased 


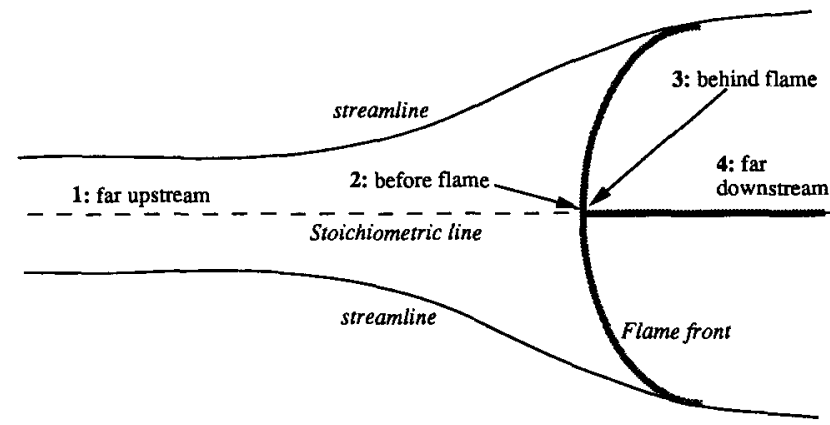

FIG. 10. Triple flame for large mixing thicknesses. Propagation speed can be determined using conservation relations along the stoichiometric (dashed) line at stations 1-4, and also in the stream tube traced by the thin solid lines.

the propagation speed increases. We expect that for very large mixing thicknesses, where $\delta_{M} / \delta_{L}^{0} \gg 1$, the flame speed will reach some asymptotic value.

An estimate of the flame speed can be derived by considering conservation relations applied to several locations along the stoichiometric line, shown in Fig. 10. These locations are (1) far upstream, (2) immediately preceding the flame, (3) immediately following the flame, and (4) far downstream. For large mixing thicknesses, the flow in the immediate vicinity of the flame is nearly one dimensional. Thus, between stations (2) and (3) we can apply the Rankine-Hugoniot relations:

$$
\begin{aligned}
& \rho_{2} u_{2}=\rho_{3} u_{3}, \\
& P_{2}+\rho_{2} u_{2}^{2}=P_{3}+\rho_{3} u_{3}^{2} .
\end{aligned}
$$

On either side of the flame, the density can be taken as constant,

$$
\rho_{1}=\rho_{2} ; \quad \rho_{3}=\rho_{4},
$$

so along the stoichiometric streamline,

$$
\begin{aligned}
& P_{1}+\frac{1}{2} \rho_{1} u_{1}^{2}=P_{2}+\frac{1}{2} \rho_{2} u_{2}^{2}, \\
& P_{3}+\frac{1}{2} \rho_{3} u_{3}^{2}=P_{4}+\frac{1}{2} \rho_{4} u_{4}^{2} .
\end{aligned}
$$

The flow velocity along the stoichiometric surface in front of the triple flame is the planar premixed flame speed:

$$
u_{2}=S_{L}^{0} \text {. }
$$

We are interested in determining $u_{1} / u_{2}$. In addition to the above relations, the integral conservation laws are needed. We choose a control volume that connects the upstream and downstream locations by streamlines that "touch" the edges of the premixed flames. If we denote the vertical distance between the streamlines at any location by $\delta$, then for mass conservation we have

$$
\Delta \psi=\rho_{1} u_{1} \delta_{1} \sim \rho_{4} u_{4} \delta_{4},
$$

where $\psi$ is the mass-based streamfunction:

$$
\frac{\partial \psi}{\partial x}=-\rho v ; \quad \frac{\partial \psi}{\partial y}=\rho u .
$$

As a first approximation we have assumed $u_{4}$ is constant behind the flame far downstream.

Combining these conservation relations and solving for $u_{1} / u_{2}$, we have

$$
\left(\frac{u_{1}}{u_{2}}\right)^{2} \sim \frac{\left(\rho_{1} / \rho_{4}-1\right)}{\left(P_{1}-P_{4}\right) /\left(\frac{1}{2} \rho_{1} u_{1}^{2}\right)+1-\left(\rho_{1} / \rho_{4}\right)\left(\delta_{1} / \delta_{4}\right)^{2}} .
$$

Equation (6) thus provides the propagation speed relative to the planar flame speed as a function of the density, pressures, and thicknesses at stations (1) and (4). However, we would like to have this expression in terms of the densities alone. Therefore, we need an integral form for momentum conservation over the control volume. If we denote the average pressure along the control volume streamlines as $P_{s}$, then the global momentum relation is

$$
P_{1} \delta_{1}+\rho_{1} u_{1}^{2} \delta_{1}+P_{s}\left(\delta_{4}-\delta_{1}\right)=P_{4} \delta_{4}+\rho_{4} u_{4}^{2} \delta_{4} .
$$

For an unconfined flow we can make the assumption of $P_{1}=P_{4}$. The difficulty now is that we need to obtain $P_{s}$, the pressure distribution along the streamline. In general, this pressure varies as one passes near the flame, however, as a first approximation we can take $P_{s}=P_{1}$. Using this assumption, Eq. (7) gives

$$
\rho_{1} u_{1}^{2} \delta_{1} \sim \rho_{4} u_{4}^{2} \delta_{4},
$$

which, after using the overall mass conservation equation, Eq. (5) becomes

$$
u_{1} \sim u_{4} ; \quad \frac{\delta_{1}}{\delta_{4}} \sim \frac{\rho_{4}}{\rho_{1}} .
$$

Substituting this into Eq. (6), along with the equal pressure assumption, gives

$$
\frac{U_{F}}{S_{L}^{0}}=\frac{u_{1}}{u_{2}} \sim\left(\frac{\rho_{1}}{\rho_{4}}\right)^{1 / 2} \text {. }
$$

Numerically we cannot simulate flames with the length scale ratios required in the above formulation; however, we do observe the scaling behavior in Eq. (8) for our simulations with large mixing thicknesses, shown in Fig. 11. At this point we should comment on the errors incurred by using the constant pressure assumption along the streamlines defining our control volume. Along the centerline the pressure rises before the flame, drops across the flame, and then rises behind the flame. This horizontal pressure variation becomes weaker at vertical locations away from the centerline. Recall that the control volume is bounded laterally by streamlines that are closer to the centerline in the unburned flow and farther away from the streamlines in burned flow. As a result, the error in the constant pressure assumption is more pronounced in front of the flame. A correction to this error would then exhibit a force directed against the propagation of the flame, reducing the flame speed.

\section{DISCUSSION AND CONCLUSIONS}

In this study we have reported on the role heat release plays in the propagation of laminar triple flames. Simulations of triple flames have shown that the primary effect of heat release is to increase the flame speed due to the flow redi- 


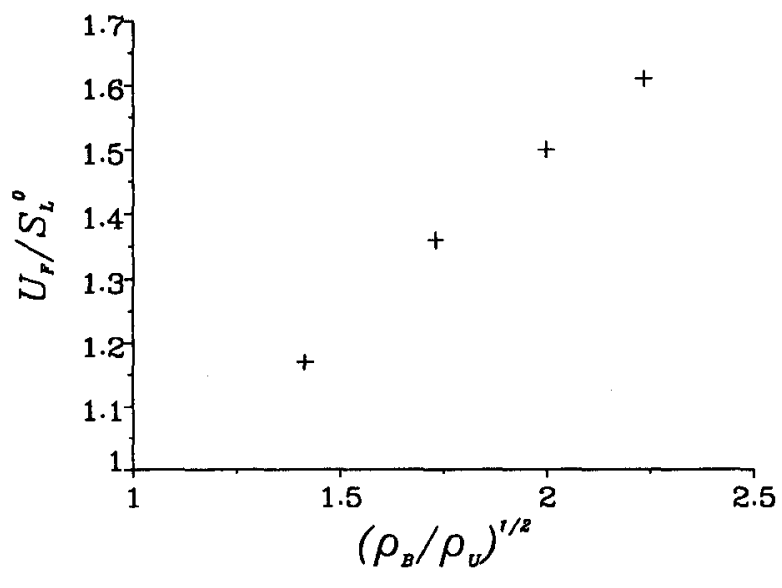

FIG. 11. Flame speed ratio versus density ratio in the simulation. Data are from simulations VI-IX.

rection that occurs in front of the flame. This redirection is a consequence of the acceleration of normal velocity across the premixed portions of the flame. Having established this as the basic mechanism for different propagation speeds relative to the zero heat release case, we have also investigated how this mechanism is affected by physical parameters.

The effect of the mixture fraction gradient, or alternatively the mixing thickness, on the flame speed was also investigated. Changing the mixing thickness modifies the amount of burning that occurs in the premixed and diffusion components of the triple flame. For larger mixing thickness, more burning takes place in the two premixed sections of the flame. Any burning in these sections adds to the flow redirection, and therefore the greatest increase in flame speed occurs for the larger mixing thicknesses. For small mixing thicknesses a large percentage of the burning occurs in the diffusion flame, which does not modify the flame speed.

Quenching was not observed in these flames. The same mechanism that increases the flame propagation also reduces the mixture fraction gradient in front of the flame. In essence, heat release adds a self-preservation mechanism to triple flame. This modification of the approaching mixture fraction gradient has implications in modeling, where the scalar dissipation (square of the mixture fraction gradient) is often used as a free parameter in models of nonpremixed combustion. $^{14}$

For large mixing thicknesses, a scaling relation was pre- sented for the increase in flame speed as a function of the density drop across the flame. This half-power law provides an upper bound for the flame speed.

The present study applies to laminar flows. We can speculate that these mechanisms are also valid in turbulent flows, where a "triple-flamelet" approach can be used in the appropriate combustion regime. However, the degree to which these model flames describe flame stabilization in turbulence has yet to be ascertained.

\section{ACKNOWLEDGMENTS}

This research is supported by the Center for Turbulence Research. The authors would like to thank T. Poinsot and D. Veynante for their input during the CTR 1994 Summer Program, and also the helpful discussions and perspicacious comments by T. Mantel and J.-M. Samaniego.

${ }^{1}$ A. Liñán, "Ignition and flame spread in laminar mixing layers," in Combustion in High Speed Flows, edited by J. Buckmaster, T. L. Jackson, and A. Kumar (KJuwer Academic, Boston, 1994), p. 461.

${ }^{2}$ J. Réveillon, P. Domingo, and L. Vervisch, "Non-premixed flame ignition in turbulent flows, triple flames," Tenth Symposium on Turbulent Shear Flows, University Park, PA, 14-16 August 1995.

${ }^{3} \mathrm{~N}$. Peters, "The modeling of combustion and pollutant formation in engine flows," in Conference on Unsteady Combustion, Espinko, Portugal, 1993.

${ }^{4} \mathrm{H}$. Phillips, "Flame in a buoyant methane layer," in 10th International Symposium on Combustion, Cambridge, 1965, p. 1277.

${ }^{5}$ P. N. Kioni, B. Rogg, K. N. C. Bray, and A. Linán, "Flame spread in laminar mixing layers: The triple flame," Combust. Flame 95, 276 (1993).

${ }^{6} \mathrm{~J}$. W. Dold, "Flame propagation in a nonuniform mixture: Analysis of a slowly varying triple flame," Combust. Flame 76, 71 (1989).

${ }^{7}$ J. W. Dold, L. J. Hartley, and D. Green, "Dynamics of laminar tripleflamelet structures in non-premixed turbulent combustion," in Dynamical Issues in Combustion Theory, The IMA Volumes in Mathematics and its Applications, edited by P. C. Fife, A. Linán, and F. A. Williams (SpringerVerlag, New York, 1991), pp. 83-105.

${ }^{8} \mathrm{~L}$. J. Hartley and J. W. Dold, "Flame propagation in a nonuniform mixture: Analysis of a triple flame," Combust. Sci. Technol. 80, 23 (1991).

${ }^{9}$ P. A. McMurtry, J. J. Riley, and R. W. Metcalfe, "Effects of heat release on the large-scale structure in turbulent mixing layers," J. Fluid Mech. 199, 297 (1989).

${ }^{10}$ F. A. Williams, Combustion Theory (Addison-Wesley, New York, 1986)

${ }^{11}$ S. K. Lele, "Compact finite difference schemes with spectral-like resolution," J. Comput. Phys. 103, 16 (1992).

${ }^{12} \mathrm{~T}$. Poinsot and S. K. Lele, "Boundary conditions for direct numerical simulations of compressible viscous flows," J. Comput. Phys. 101, 104 (1992).

${ }^{13}$ A. K. Kerstein, W. T. Ashurst, and F. A. Williams, "Field equation for interface propagation in an unsteady homogeneous flow field," Phys. Rev. A 37, 2728 (1988).

${ }^{14}$ N. Peters, "Local quenching due to flame stretch and non-premixed turbulent combustion," Combust. Sci. Technol. 30, 1 (1983). 